\title{
BIOECOLOGÍA Y PESQUERÍA DE Pseudoplatystoma fasciatum (LINNAEUS, 1766; PISCES), DONCELLA, EN LA REGIÓN UCAYALI
}

\author{
BIOECOLOGY AND FISHERY OF Pseudoplatystoma fasciatum (LINNAEUS, 1766; PISCES), \\ DONCELLA, INTHE UCAYALI REGION
}

\author{
Sonia A. Deza T. ${ }^{1}$, Róger S. Bazán A. ${ }^{2}$ y Zoila G. Culquichicón M. ${ }^{3}$
}

\begin{abstract}
RESUMEN
El estudio se desarrolló durante el periodo de febrero de 2001 a febrero de 2002. Se describen aspectos bioecológicos como hábitos alimentarios, biología reproductiva, relación peso-longitud y algunos aspectos pesqueros. La Pseudoplatystoma fasciatum, doncella, presenta hábitos alimentarios carnívoros teniendo al Prochilodus nigricans, boquichico, como una de las especies más importantes en su alimentación. Su proporción sexual global fue favorable a las hembras (1.51:1); éstas predominan a tallas mayores y los machos a tallas menores. La talla media de madurez es $93.92 \mathrm{~cm}$ de longitud total en hembras y de $78.49 \mathrm{~cm}$ en machos. El desove se produce a los 102.24 y 87.89 cm en hembras y machos respectivamente; la época de reproducción de esta especie está comprendida entre los meses de noviembre y marzo, su crecimiento es isométrico. Con relación a su pesquería, la producción total durante el 2001 fue de 1857.6 t llegando a ocupar el segundo lugar en los desembarques. Las principales zonas de pesca son Sheshea en el alto Ucayali y Santa Isabel en el bajo Ucayali. Las artes de pesca más empleadas son las redes tramperas, sin embargo en la vaciante las más efectivas por su mayor producción son las redes rastreras.
\end{abstract}

Palabras clave: región Ucayali, doncella, Pseudoplatystoma fasciatum, bioecología, pesquería, acuicultura.

\begin{abstract}
The study was carried out during the period from February 2001 to February 2002. Bioecology aspects as alimentary habits, reproductive biology, length-weight relationship and some characteristics of the fishery were described. The Pseudoplatystoma fasciatum, doncella, presents carnivorous habits having the Prochilodus nigricans, boquichico, as one of the most important species in its diet. The global sexual proportion was favourable the females (1.51:1), they were predominant at bigger sizes and the males at smaller sizes. The mean size of sexual maturity was $93.92 \mathrm{~cm}$ of total length in females and $78.49 \mathrm{~cm}$ in males. This specie spawns at 102.24 $\mathrm{cm}$ and 87.89 in females and males respectively. The reproduction season of this specie is between November and March, its growth is isometric. Related to the fishery, the total production for 2001 was $1857.6 \mathrm{t}$, being the second species in the landings. The main fishery areas are Sheshea in higher Ucayali river and Santa Isabel in lower Ucayali river. The most employed fishing gears are the trap nets, however, during the decrease of the level of waters (vaciante) the most effective is trawling, nest resulting in higher production the trawl nets.
\end{abstract}

Key words: Ucayali region, doncella, Pseudoplatystoma fasciatum, bioecology, fishery, aquaculture.

\section{INTRODUCCIÓN}

Pseudoplatystoma fasciatum, es una especie perteneciente a la familia Pimelodidae, que forma parte de los grandes bagres de la Amazonía; de hábitos alimenticios carnívoros (Goulding, 1980) y de gran importancia

1 Instituto de Investigaciones de la Amazonía Peruana - IIAP. Programa de Ecosistemas Acuáticos. km 12.4 de la carretera Federico Basadre. Correo electrónicol: iiapu@terra.com.pe , soniaamparo41@hotmail.com , Pucallpa-Perú

2 Universidad Nacional de Trujillo. Facultad de Ciencias Biológicas. Escuela de Biología Pesquera. San Martín 761. Correo electrónico: neyarose@hotmail.com , Pucallpa-Perú

3 Universidad Nacional de Trujillo. Correo Electrónico: zculquichicón@pg.unitru.edu.pe ,zgculq@chanchan.unitru.edu.pe 
comercial en la Amazonía colombiana (Salinas et al. 1999), en la Amazonía peruana (Pucallpa e Iquitos), así como en la cuenca del río Guaporé (Barthem et al. 1995) y en la cuenca del río Mamore en Bolivia (Lauzanne et al. 1990).

En los últimos cinco años la extracción de esta especie en la región Ucayali se ha incrementado, debido a la gran demanda de su carne para los exportadores (Colombia y Brasil), originándose una fuerte explotación, que según las estadísticas pesqueras la sitúan en el segundo lugar después del boquichico. Sin embargo son muy pocos los estudios realizados en esta región, que aporten como estrategias de manejo de las poblaciones para asegurar una producción sostenida a través del tiempo. Los pocos trabajos existentes están referidos a su biología, taxonomía y la pesca comercial en algunos países sudamericanos, pero ninguno trata profundamente su biología (Goulding, 1980; Smith, 1981).

En este sentido, con el propósito de brindar información para futuras estrategias tendentes a su regulación pesquera, se tuvo como objetivos el determinar el hábito alimentario, los aspectos reproductivos y su relación con los factores ambientales que influyen en el comportamiento de esta especie, así como también el análisis de su pesquería en esta región.

\section{MATERIAL Y MÉTODO}

El material estudiado fue obtenido mediante muestreos al azar en los puertos de desembarque y mercados de Pucallpa, realizándose muestreos biométricos a 1606 especímenes y el análisis biológico a 1358 en la estación experimental del Instituto de Investigaciones de la Amazonía Peruana (IIAP) durante el periodo de febrero de 2001 a febrero de 2002. Para el análisis de su pesquería, se utilizaron datos pesqueros del año 2001, información que fue proporcionada por la Dirección Regional de Pesquería de Ucayali.

Para cada espécimen se registró la longitud total (Lt) y a la horquilla (Lh) en centímetros, usando una cinta métrica Fiber Glass de $1.5 \mathrm{~m}$ graduada al milímetro, y el peso total usando una balanza romana de $20 \mathrm{~kg}$ con una sensibilidad de $0.5 \mathrm{~kg}$; también se registró el sexo, grado de madurez, peso gonadal, peso de estómago lleno y vacío, usando una balanza semianalítica Sartorius Gottingen modelo BL310 de 310 g y con una sensibilidad de $0.01 \mathrm{~g}$.

\subsection{BIOECOLOGÍA}

\subsubsection{Hábitos alimentarios}

Fueron determinados mediante el análisis del contenido estomacal, para lo cual se analizaron 493 estómagos de doncella; las especies presas encontradas, fueron identificadas hasta el nivel de especie y las que no fueron posibles, se identificaron sólo hasta familia. Las especies que se presentaron por una sola vez, fueron incluidas en la categoría «miscelánea». También se realizó el análisis cuantitativo utilizando los métodos: numérico, estimación porcentual, frecuencia de ocurrencia y gravimétrico descritos por Tresierra \& Culquichicón (1993).

Asimismo, se calculó el índice de vacuidad según fórmula descrita por Vegas (1987):

$$
\mathrm{V}=(\mathrm{Ev} / \mathrm{N}) * 100
$$

Donde:

$$
\begin{aligned}
& \mathrm{V}=\text { coeficiente de vacuidad } \\
& \mathrm{Ev}=\text { estómagos vacíos } \\
& \mathrm{N}=\text { número de estómagos analizados }
\end{aligned}
$$




\subsection{BIOLOGÍA REPRODUCTIVA}

\subsubsection{Proporción sexual}

Fueron utilizados 1358 especímenes de doncella para determinar la proporción sexual global, dividiendo el número de hembras entre el número de machos. Para calcular la proporción sexual a la talla, se elaboró una tabla de frecuencias de tallas, usándose la longitud total, con una amplitud interval de cinco cm, calculándose la proporción sexual para cada intervalo. Luego se calculó el porcentaje de machos y de hembras, para posteriormente graficar el porcentaje versus la talla. Para determinar las diferencias significativas de la relación 1:1 se aplicó el test estadístico del Chi cuadrado (X²), según Zar (1974):

$$
\mathrm{X}^{2}=\Sigma\left((\mathbf{O}-\mathbf{E}-0.5)^{2} / \mathbf{E}\right)
$$

Donde:

$$
\begin{array}{ll}
\mathrm{X}^{2} & =\text { chi cuadrado } \\
0 & =\text { frecuencia observada } \\
\mathrm{E} & =\text { frecuencia esperada }
\end{array}
$$

\subsubsection{Estado de madurez sexual y época de desove}

El análisis del estado de madurez sexual se realizó mediante la observación directa de las gónadas en el momento de la evisceración de los especímenes muestreados, utilizándose una escala macroscópica empírica de seis estadios descrita por García et al. (1998).

La época de desove fue determinada mediante el índice gónado somático (IGS), el cual se calculó para hembras y machos mensualmente, usando la siguiente formula:

$$
\text { IGS }=\text { PG }(\mathrm{g}) * 100 / \text { P. eviscerado }
$$

Donde:

$$
\begin{aligned}
& \text { PG }=\text { peso de las gónadas } \\
& \text { Pevis }=\text { peso corporal eviscerado }
\end{aligned}
$$

\subsubsection{Talla media de madurez y de desove}

Para calcular la talla media de madurez (TMM) y la talla media de desove (TMD), se elaboraron tablas de frecuencia de talla, agrupando los especímenes en estadios II y III en caso de la TMM y especímenes en estadios IV y V para la TMD; luego se calculó la frecuencia acumulada porcentual, para posteriormente graficarla versus la talla.

\subsubsection{Relación entre el ciclo hidrológico, precipitaciones y temperatura con el patrón de reproducción}

Para determinar la relación entre el ciclo hidrológico, precipitaciones y temperatura con el patrón de reproducción de doncella, se recolectaron mensualmente datos de: nivel del río Ucayali, precipitaciones y temperatura, los cuales fueron proporcionados por el Ministerio de Transportes y Comunicaciones (MTC) a través de la oficina de Transporte Acuático en el caso de los niveles y del Ministerio de Agricultura a través de la Dirección de Información Agraria (DIA-CORPAC) en caso de las precipitaciones y temperaturas. Estos datos fueron graficados frente a los valores del IGS. 


\subsection{RELACIÓN PESO-LONGITUD}

Para determinar este parámetro, se usaron los pesos y las longitudes totales, aplicándose la siguiente fórmula:

$$
\mathbf{P}=\mathbf{F} \mathbf{L}^{\mathbf{n}}
$$

Donde:

$$
\begin{aligned}
& \mathrm{P}=\text { es el peso total } \\
& \mathrm{L}=\text { es la longitud total } \\
& \mathrm{F}=\text { es la constante (intercepto) } \\
& \mathrm{n}=\text { es el coeficiente (pendiente) }
\end{aligned}
$$

\subsection{PESQUERÍA}

Los volúmenes de desembarque de doncella fueron obtenidos de las estadísticas proporcionadas por la Dirección Regional de Pesquería de Ucayali, registrándose además las zonas de captura que están ubicadas en el alto y bajo Ucayali. Las artes y aparejos de pesca fueron registrados anotándose sus características.

Para el cálculo de la captura por unidad de esfuerzo (CPUE) se utilizaron los desembarques al estado fresco y congelado, mediante la siguiente fórmula:

\section{CPUE $=\mathrm{C} / \mathrm{f}$}

Donde:

$$
\begin{aligned}
& \mathrm{C}=\text { capturas }(\mathrm{kg}) \\
& \mathrm{f}=\text { esfuerzo pesquero (número de viajes) }
\end{aligned}
$$

\section{RESULTADOS}

\subsection{HÁBITOS ALIMENTARIOS}

Del análisis de 493 estómagos, sólo el 20.28\% tuvieron contenido estomacal, en consecuencia, durante el periodo de estudio el índice de vacuidad, siempre fue superior a 50\%, presentando un índice de vacuidad total de 79.72.

Se identificaron 18 especies presas pertenecientes a nueve familias: boquichico a la familia Prochilodontidae; llambina, ractacara, chío chío y san pedro a Curimatidae; palometa, paña roja y paña blanca a Serrasalmidae; sardina y mojarra a Characidae; chambira a Cynodontidae; bagre, rego rego y poyi poyi a Doradidae; shitari y loricarido a Loricariidae; shiruy a Callichthydae y corvina que pertenece a la familia Sciaenidae.

Los métodos cuantitativos, ubican al boquichico en primer lugar, seguido por el bagre y la palometa (cuadro 1). Sin embargo el análisis a través del tiempo, nos indica cambios en la importancia de la especie presa (cuadro 2). 
Cuadro 1. Porcentajes obtenidos por los diferentes métodos para la determinación de las especies presas principales de Pseudoplatystoma fasciatum, doncella (febrero 2001 a febrero 2002).

\begin{tabular}{|c|c|c|c|c|c|c|}
\hline \multirow{2}{*}{ Especie } & \multirow{2}{*}{$\begin{array}{c}\text { Frecuencia } \\
\text { (n) }\end{array}$} & \multirow{2}{*}{$\begin{array}{c}\text { Peso } \\
\text { (g) }\end{array}$} & \multicolumn{4}{|c|}{ Método } \\
\hline & & & Numérico & $\begin{array}{l}\text { Estimación } \\
\text { porcentual }\end{array}$ & $\begin{array}{l}\text { Frecuencia } \\
\text { ocurrencia }\end{array}$ & Gravimétrico \\
\hline Boquichico & 33 & 2758.39 & 29.20 & 34.15 & 28 & 44.95 \\
\hline Bagre & 29 & 1176.62 & 25.66 & 17.68 & 15 & 19.17 \\
\hline Corvina & 2 & 110.00 & 1.77 & 1.22 & 1 & 1.79 \\
\hline Chío chío & 7 & 214.44 & 6.19 & 4.88 & 4 & 3.49 \\
\hline Llambina & 7 & 324.81 & 6.19 & 4.88 & 4 & 5.29 \\
\hline Mojarra & 2 & 42.20 & 1.77 & 2.44 & 2 & 0.69 \\
\hline Palometa & 13 & 739.94 & 11.50 & 12.80 & 11 & 12.06 \\
\hline Shiruy & 2 & 139.74 & 1.77 & 1.22 & 1 & 2.28 \\
\hline Miscelánea & 18 & 630.99 & 15.93 & 19.51 & 16 & 10.28 \\
\hline Total & 113 & 6137.13 & 99.98 & 98.78 & 82.00 & 100.00 \\
\hline
\end{tabular}

Cuadro 2. Variación del contenido estomacal (en \%) de Pseudoplatystoma fasciatum, doncella, por épocas del año.

\begin{tabular}{|l|c|c|c|c|}
\hline \multirow{2}{*}{ Especie } & \multicolumn{4}{|c|}{ Época del año } \\
\cline { 2 - 5 } & Creciente & Interfase a vaciante & Vaciante & Interfase a creciente \\
\hline Boquichico & 61.49 & 9.32 & 91.52 & 16.74 \\
Bagre & 11.15 & 60.75 & 2.63 & 6.39 \\
Corvina & - & 1.47 & - & - \\
Chío chío & - & - & - & 42.27 \\
Llambina & - & 0.77 & 0.84 & 3.30 \\
Palometa & 8.17 & 7.61 & 5.01 & 7.48 \\
Shiruy & - & 0.67 & - & - \\
Miscelánea & 19.22 & 19.41 & - & 23.82 \\
\hline Total & $\mathbf{1 0 0 . 0 0}$ & $\mathbf{1 0 0 . 0 0}$ & $\mathbf{1 0 0 . 0 0}$ & $\mathbf{1 0 0 . 0 0}$ \\
\hline
\end{tabular}

\subsection{BIOLOGÍA REPRODUCTIVA}

\subsubsection{Proporción sexual}

Respecto a la proporción sexual global, las hembras presentan un dominio. Durante la creciente y la interfase a creciente el dominio es mayor. Pero en las épocas de interfase a vaciante y vaciante, la proporción de hembras disminuye hasta alcanzar una proporción cercana a 1:1, sin considerar la estacionalidad, la proporción sexual es diferente de la relación 1:1 ( $\left.\mathrm{x}^{2}=56.09, \mathrm{p}<0.05\right)$; aceptándose la proporción 2:1 (1.5:1) (cuadro 3). El análisis de la proporción sexual a la talla, indica que las hembras predominan a tallas mayores, que van desde los 82 a $127 \mathrm{~cm}$ de longitud total y los machos a tallas menores de 37 a $77 \mathrm{~cm}$. 
Cuadro 3. Proporción sexual global de doncella y prueba de Chi cuadrado por épocas del año.

\begin{tabular}{|c|c|c|c|c|c|c|c|c|}
\hline \multirow[b]{2}{*}{ Épocas del año } & \multicolumn{2}{|c|}{ Número } & \multirow[t]{2}{*}{ Total } & \multicolumn{2}{|c|}{ Porcentaje } & \multirow{2}{*}{$\begin{array}{c}\text { Proporción } \\
\text { sexual } \\
\text { H:M }\end{array}$} & \multirow{2}{*}{$\begin{array}{c}\mathrm{Chi}^{2} \\
\text { calculado }\end{array}$} & \multirow{2}{*}{$\begin{array}{c}\text { Grado de } \\
\text { significancia } \\
\text { Chi' tab }=\mathbf{3 . 8 4}\end{array}$} \\
\hline & $\mathbf{H}$ & M & & $\mathbf{H}$ & $\mathbf{M}$ & & & \\
\hline Creciente (2001) & 77 & 29 & 106 & 72.64 & 27.36 & $2.65: 1$ & 21.75 & Muy significativo \\
\hline Interfase a vaciante & 231 & 186 & 417 & 55.40 & 44.60 & $1.24: 1$ & 4.86 & Significativo \\
\hline Vaciante & 278 & 205 & 483 & 57.56 & 42.44 & $1.36: 1$ & 11.04 & Significativo \\
\hline Interfase a creciente & 137 & 80 & 217 & 63.13 & 36.87 & $1.71: 1$ & 14.98 & Significativo \\
\hline Creciente (2002) & 94 & 41 & 135 & 69.63 & 30.37 & $2.29: 1$ & 20.81 & Muy significativo \\
\hline Total & 817 & 541 & 1358 & 60.16 & 39.84 & $1.51: 1$ & 56.09 & Muy significativo \\
\hline
\end{tabular}

$\mathrm{H}$ : hembras

M: machos

\subsubsection{Estado de madurez sexual y época de desove}

La presencia de individuos inmaduros y en desarrollo se da en casi todos los meses, recién en octubre aparecieron especímenes con estadios III y el aumento progresivo de la maduración de noviembre a febrero en caso de la hembras y a marzo en caso de los machos.

El periodo reproductivo se inicia en noviembre y se prolonga hasta marzo. En noviembre, el IGS supera el 0.50\% alcanzado un valor de $0.70 \%$, lo que indica el inicio de la maduración sexual. En febrero, el IGS evoluciona hasta un valor máximo de $4.30 \%$, luego se produce su descenso, que marca el inicio del periodo de desove. Este último concluye en el mes de abril cuando se registra un IGS de $0.36 \%$, que se mantiene estacionario hasta octubre (figura 1).

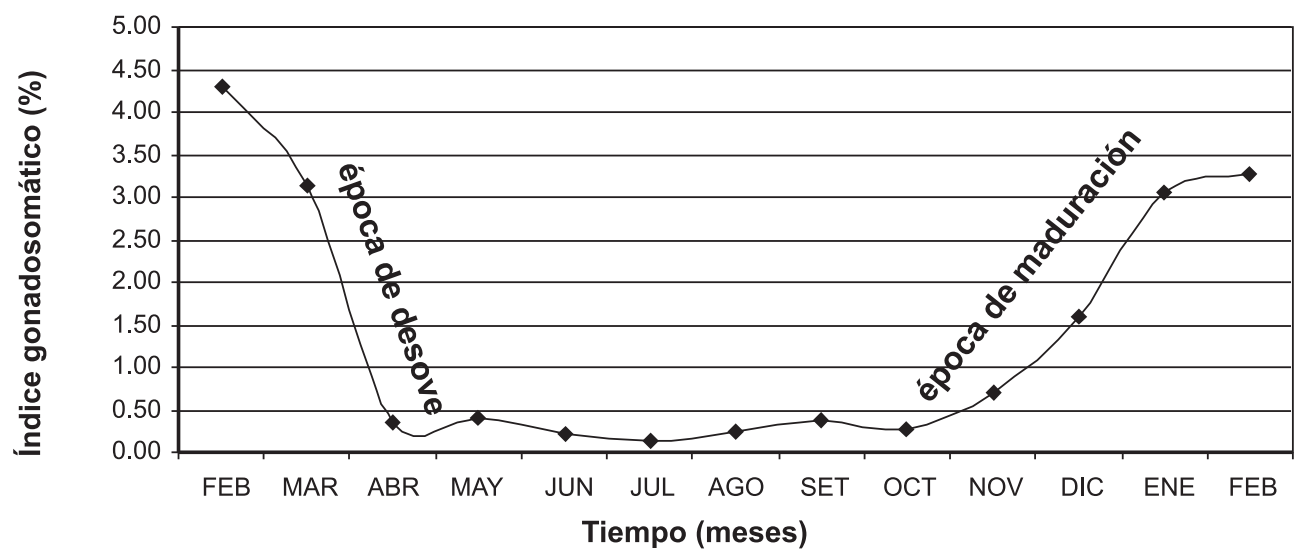

Figura 1. Época de maduración y desove de Pseudoplatystoma fasciatum, doncella. 


\subsubsection{Talla media de madurez y de desove}

La doncella alcanza la talla media de madurez a una longitud total de $93.92 \mathrm{~cm}$ en hembras y de $78.49 \mathrm{~cm}$ en machos, mientras que la talla media de desove se produce a los 102.24 y $87.89 \mathrm{~cm}$ de longitud total en hembras y machos respectivamente (figura 2).

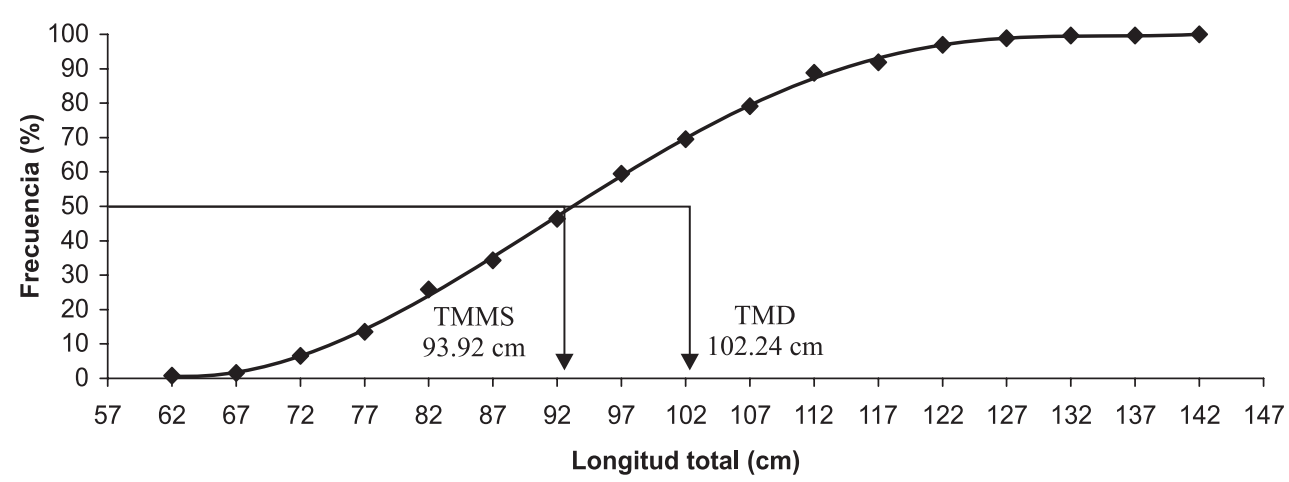

(a)

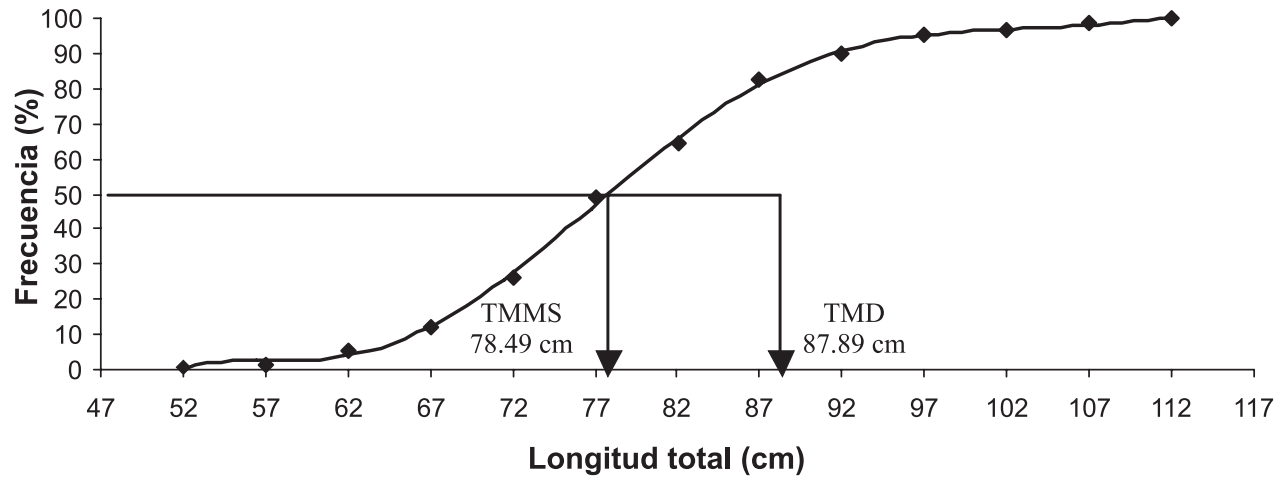

(b)

Figura 2. Talla media de madurez (TMM) y talla media de desove (TMD) en hembras (a) y machos (b) de Pseudoplatystoma fasciatum, doncella.

\subsubsection{Relación entre el ciclo hidrológico, precipitaciones y temperatura con el patrón de reproducción}

La relación entre el ciclo hidrológico, las precipitaciones y la temperatura de la región Ucayali con el patrón de reproducción de doncella, indica que el desove de esta especie está vinculado al aumento del nivel del río antes de que éste alcance su máximo nivel (figura 3a). Las relaciones con las precipitaciones y la temperatura no están muy claras, especialmente con las precipitaciones (figuras 3b y 3c). 


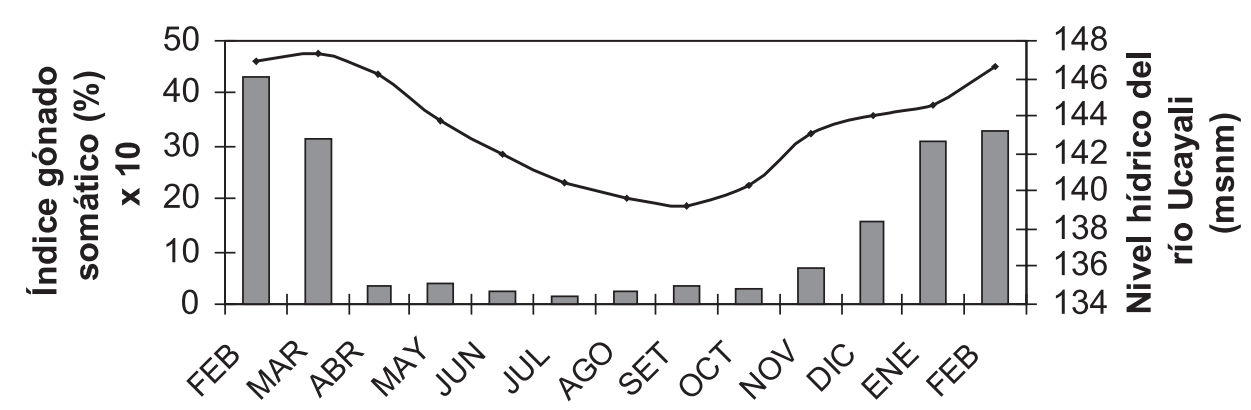

Tiempo (meses)

$\square$ IGS $\rightarrow$ Nivel

(a)

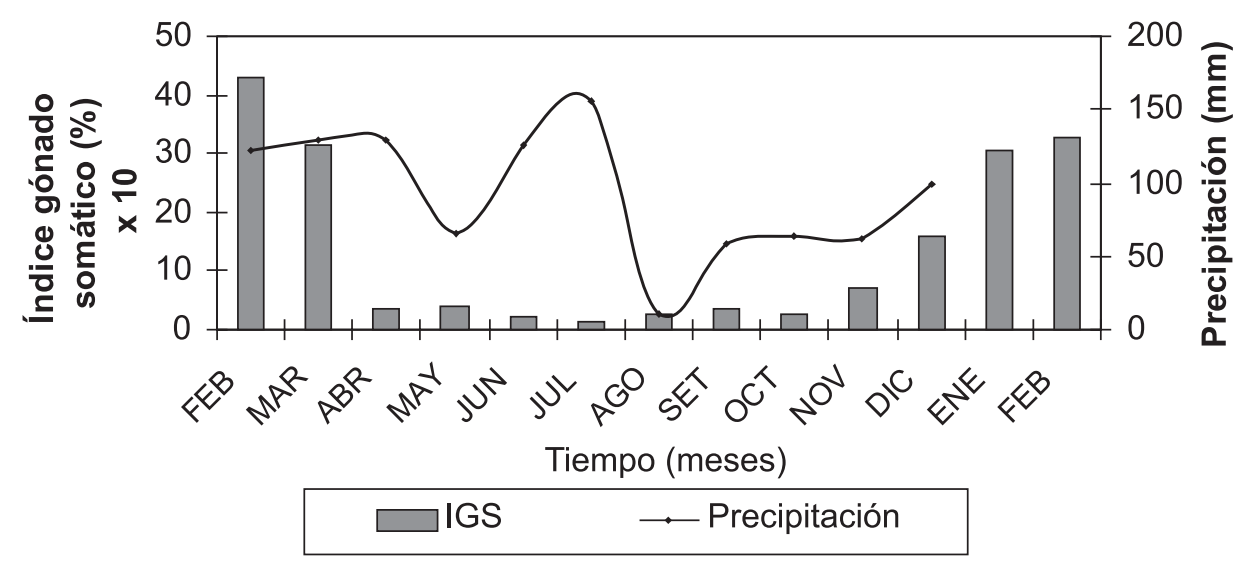

(b)

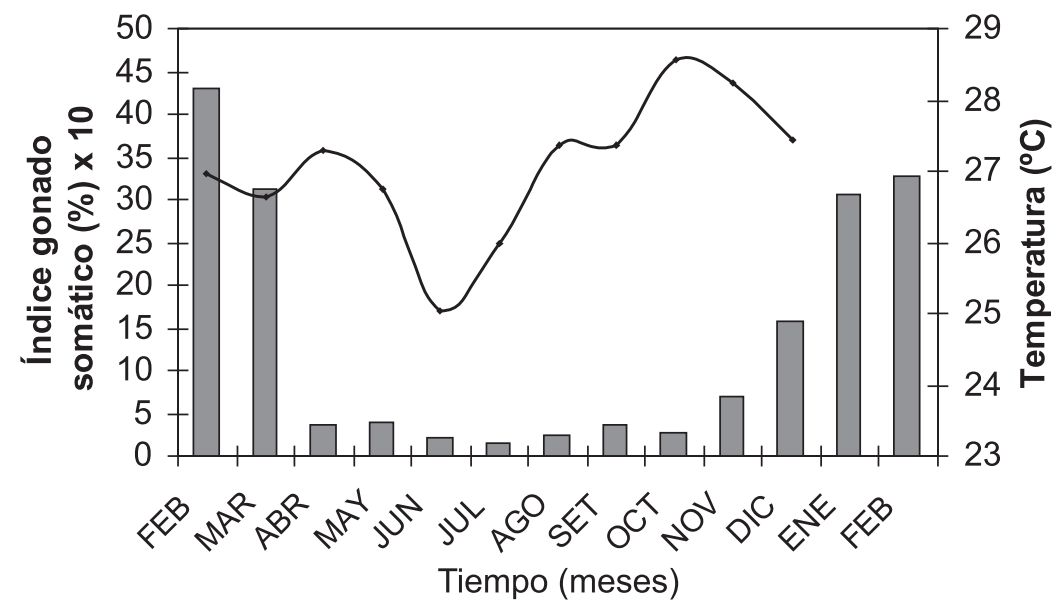

$\square$ IGS $\rightarrow$ Temperatura

(c)

Figura 3. Relación entre el patrón reproductivo y: a) nivel hídrico del río Ucayali b) precipitaciones y c) temperatura 


\subsection{RELACIÓN PESO-LONGITUD}

Los especímenes muestreados mostraron un crecimiento tendiente a la isometría, como lo demuestran las ecuaciones (tipo potencial), para hembras, machos y ambos sexos. Los valores de $\mathrm{n}$ (exponente) para los tres casos son cercanos a tres.

Las ecuaciones obtenidas fueron:

$$
\begin{array}{lll}
\mathrm{H}: & \mathrm{W}_{\mathrm{t}}=0.0058 \mathrm{~L}_{\mathrm{t}}^{3.0683} & \mathrm{R}=0.98 \\
\mathrm{M}: & \mathrm{W}_{\mathrm{t}}=0.0098 \mathrm{~L}_{\mathrm{t}}^{2.8835} & \mathrm{R}=0.94 \\
\mathrm{C}: & \mathrm{W}_{\mathrm{t}}=0.006 \mathrm{~L}_{\mathrm{t}}^{3.0602} & \mathrm{R}=0.97
\end{array}
$$

\subsection{PESQUERÍA}

La producción pesquera registrada en la región Ucayali en el año 2001 asciende a 11114.5 t que comprende a 63 especies de consumo humano directo. Para la doncella la producción fue de 1857.6 t ubicándola en el segundo lugar después del boquichico del total de las especies capturadas. La mayor captura de doncella ocurrió en el mes de agosto con 308.99 t y la menor se registró en diciembre con 98.76 t (figura 4), los picos de mayor captura se registraron durante la vaciante, específicamente entre agosto y octubre.

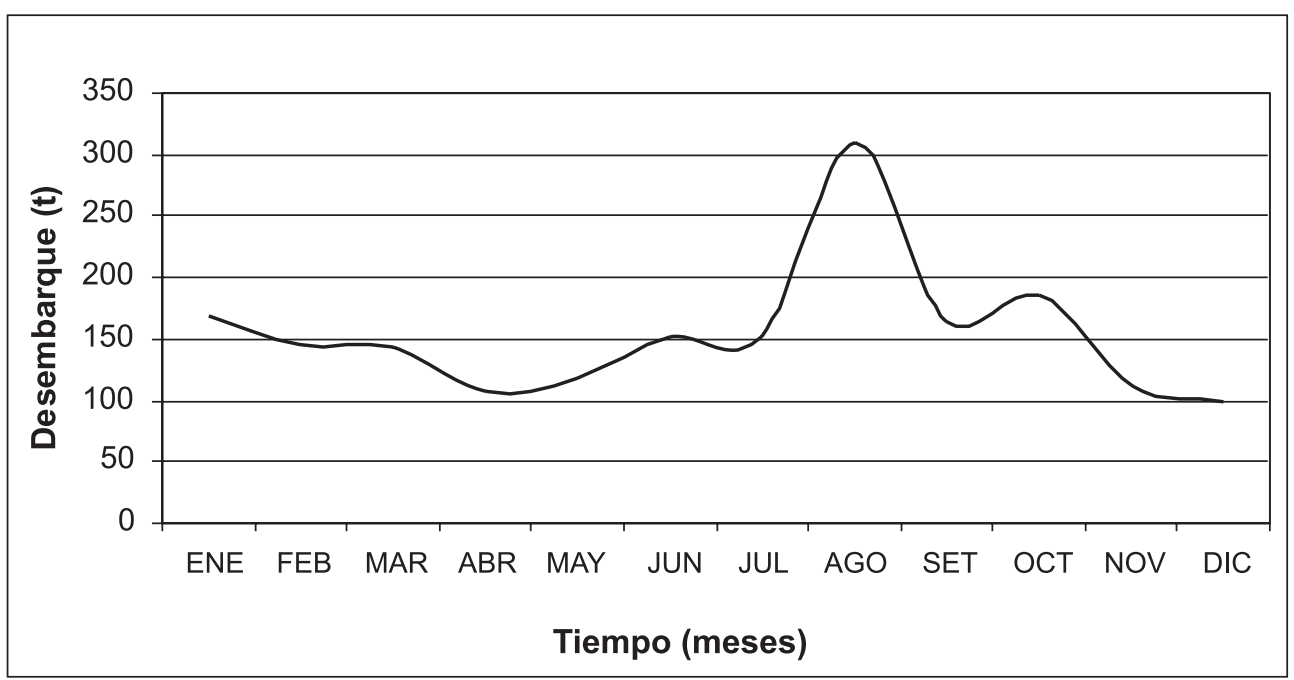

Figura 4. Evolución de los desembarques de Pseudoplatystoma fasciatum, doncella, durante el 2001 en la región Ucayali.

La captura de doncella proveniente del alto Ucayali representó el 71.84\% del desembarque total registrado, mientras que del bajo Ucayali el 28.16\%. Fueron identificadas 74 zonas de pesca, de las cuales 53 están ubicadas en el alto Ucayali y 21 en el bajo Ucayali, siendo Sheshea (alto Ucayali) y Santa Isabel (bajo Ucayali) las principales zonas por su mayor producción de este recurso pesquero. 
La captura comercial de la doncella se realiza a través de diferentes tipos de artes de pesca y aparejos como tramperas, honderas (redes agalleras a la deriva), rastreras (redes de encierre de jareta) y anzuelos. El arte más frecuente en la captura de este recurso es la red trampera, la cual predomina en la época de la creciente junto con los anzuelos. El uso de la red rastrera se limita a la época de vaciante y comienzos de la interfase a creciente. Las mayores producciones en épocas de creciente se obtienen con las redes tramperas mientras que en la vaciante y principios de la interfase a creciente las redes rastreras son las de mayor producción.

\subsubsection{Captura por unidad de esfuerzo (CPUE)}

Los valores de CPUE, durante los primeros meses fueron muy variables y bajos, en los meses finales este parámetro aumentó su valor (figura 5).

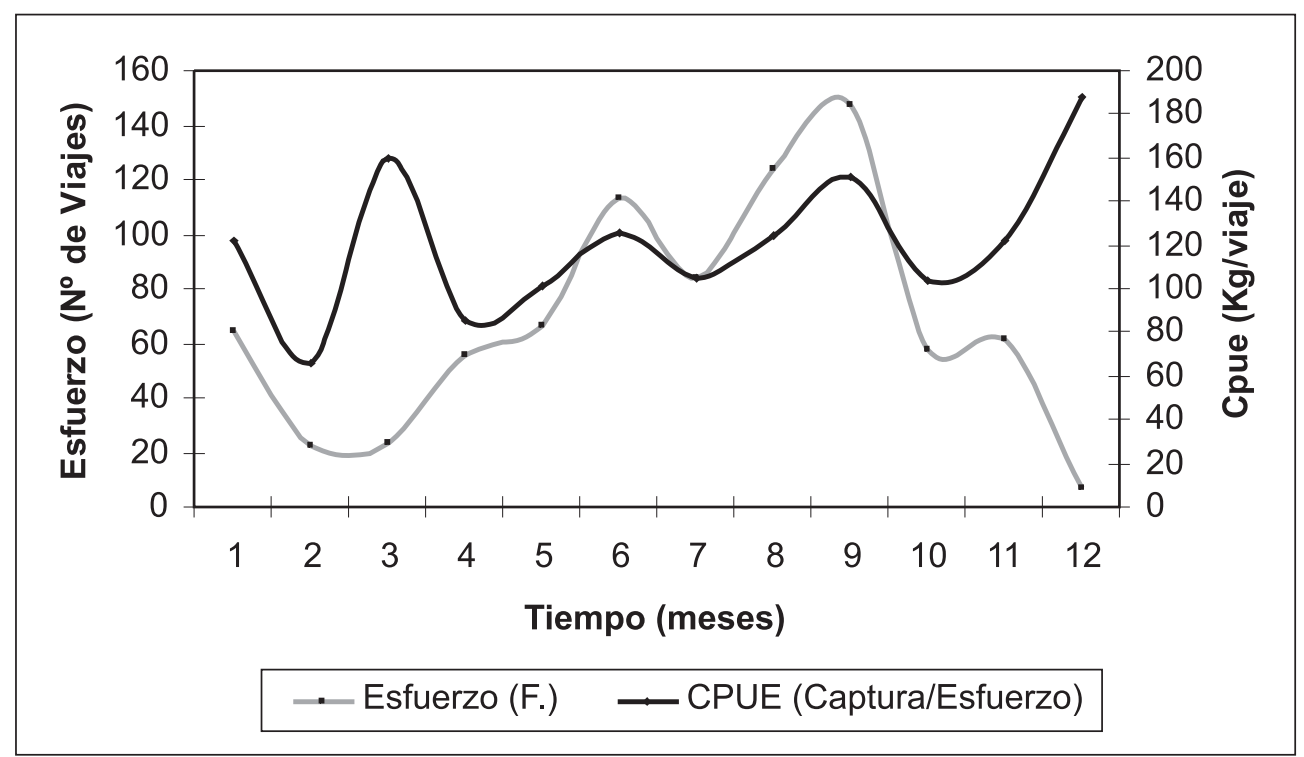

Figura 5. Captura por unidad de esfuerzo y su relación con el esfuerzo de pesca para doncella desembarcada en la región Ucayali. 


\section{DISCUSIÓN}

La $P$. fasciatum, doncella, se presenta como una especie de hábitos alimentarios carnívoros; la presencia constante y única de peces en los contenidos estomacales a lo largo de todo el periodo de estudio, evidencia que no hay un cambio temporal del hábito alimentario y que sugiere que la doncella es piscívora. El P. nigricans, boquichico, fue la especie más representada en los contenidos estomacales, coincidiendo con los resultados de Contreras (1997), quien ubica a la doncella como carnívoro, mencionando que éste tiene como principal especie presa al boquichico, seguido por el bagre Pimelodus sp. lo cual es reconfirmado por el presente estudio, en donde el boquichico ocupa el primer lugar como especie presa. Sin embargo el estudio detallado en el tiempo, indica que la doncella presenta cambios en lo referente a su presa principal, esto indicaría que tiene un espectro amplio de ítem alimentarios que están constituidos por diferentes especies ícticas.

$\mathrm{Al}$ analizar la proporción sexual, las hembras presentaron un dominio sobre los machos. En el invierno (interfase de creciente y la creciente) las hembras exceden a los machos, pero en verano (interfase de vaciante y en la vaciante) la proporción tiende a acercarse a 1:1, siendo el promedio de la proporción hembra-macho para todo el periodo de estudio igual a 1.51:1. Al respecto Contreras (1997) reportó una proporción sexual global de 1.9:1, favorable a las hembras, quedando establecida la predominancia de las hembras, sin embargo al confrontar los resultados mensualmente, Contreras señala para el mes de octubre una proporción sexual global favorable a los machos, hecho que nunca ocurrió en el presente estudio. Tresierra y Culquichicon (1993) y Vazzoler (1996), indican que los factores que hacen variar la proporción sexual global de 1:1 es la diferente vulnerabilidad por sexo y la mortalidad diferencial. El recurso estudiado presenta una relación hembra-macho favorable a las hembras en el invierno; este hecho se debe precisamente a la diferente vulnerabilidad por sexo, puesto que con la crecida de los ríos, el hábitat de los peces tiende a ampliarse, lo que significa que las artes de pesca se vuelven menos efectivas usándose con mayor frecuencia las redes tramperas y los anzuelos, los cuales capturan ejemplares grandes, constituidos mayormente por las hembras.

Contreras (1997) indica que los machos predominan a tallas pequeñas desde 45 hasta $75 \mathrm{~cm}$ de longitud total y las hembras de 80 a $125 \mathrm{~cm}$; los resultados del presente trabajo, confirman este hecho. Una posible explicación sería el rol que cumple cada grupo; por ejemplo los machos de algunas especies de silúridos y ciprínidos alcanzan tallas mayores que las hembras, debido a que el macho cumple la función de proteger a la prole; en aquellas especies donde las hembras predominan a tallas mayores, los machos sólo participan en el proceso reproductivo durante el desove al fertilizar los óvulos, además las hembras crecen más que los machos para producir una mayor cantidad de óvulos que garantice la perpetuación de la especie.

La determinación del IGS, indica la época de reproducción y específicamente señala la época de desove de los recursos pesqueros (Gupta, 1974 y 1975 en Tresierra et al. 2002). Precisamente el IGS incrementó a partir de noviembre, alcanzando su valor máximo en febrero, luego se produjo una caída paulatina, indicando el inicio del periodo de desove, el cual concluyó en los primeros días de abril cuando se registró un IGS de $0.36 \%$, que se mantuvo estacionario hasta octubre. El valor máximo del IGS para la doncella fue de $4.30 \%$, este valor comparado con el IGS de otros peces como el boquichico (IGS =15.6\%), es tres o cuatro veces menor, sin embargo parece ser suficiente para mantener su población.

La talla media de primera madurez sexual de la doncella es de $93.92 \mathrm{~cm}$ de longitud total en hembras y de $78.49 \mathrm{~cm}$ en machos. Estos resultados discrepan de aquellos encontrados por Contreras (1997), debido a que reporta una talla de $99 \mathrm{~cm}$ de longitud total para hembras y de $78 \mathrm{~cm}$ para los machos. Por otro lado, García et al. (1998) indican que las hembras y machos maduran a los $89.8 \mathrm{~cm}$ y $82.5 \mathrm{~cm}$ de longitud a la horquilla, dichos resultados también difieren de lo encontrado, debido a que estos alcanzaron su TMMS a los $85.03 \mathrm{~cm}$ y $71 \mathrm{~cm}$ Lh respectivamente. Respecto a la talla media de desove, García et al. (1998) indican que las hembras y machos expulsan sus productos sexuales a los $95.3 \mathrm{~cm}$ y $86.5 \mathrm{~cm} \mathrm{Lh}$; en el presente estudio las hembras y los machos realizaron dicha actividad a $92.06 \mathrm{~cm}$ y $80.15 \mathrm{~cm} \mathrm{Lh}$, esta diferencia puede deberse a la existencia de dos poblaciones diferentes, la primera con hábitat en el bajo Ucayali y el Amazonas y la otra en el alto Ucayali y sus afluentes. 
Welcomme (1979), Novoa \& Ramos (1982), Galvis et al. (1989), indican que la reproducción de muchos peces en los ríos tropicales es altamente estacional y que, en los ríos que presentan variaciones en su nivel a través del año, ocasionadas por regímenes hidrológicos variables, existe una sincronización entre los procesos reproductivos de la mayoría de peces y el incremento en el nivel de las aguas. Ciertamente con la doncella, se ha verificado que la duración de su época de reproducción, se prolonga desde noviembre hasta marzo con un pico máximo en febrero, este lapso coincide con el periodo de aguas altas del río Ucayali, comportamiento que también fue observado, para otras especies, por Guevara (1974), Basile-Martins (1978), Caramaschi (1979), Tello et al. (1992) citados por Barthem et al. (1995) y Montreuil et al. (2001). Así mismo Lauzanne et al. (1990) y Montreuil et al. (2001), mencionan que las estrategias de reproducción durante las aguas altas favorece mucho a las larvas y alevinos que encuentran diversos ambientes para su protección natural en las zonas inundadas, así como también adecuadas condiciones para su alimentación y desarrollo.

Es de importancia fundamental para el estudio del ciclo de vida de una especie, conocer su crecimiento en longitud y peso, puesto que poblaciones de una misma especie presentan tasas diferentes de crecimiento en peso (Vazzoler, 1981). Los resultados obtenidos en la relación peso-longitud para la doncella, nos muestra que esta especie presenta un crecimiento que tiende a la isometría, debido a que el valor de n (coeficiente de la relación peso-longitud) es igual a tres, resultados semejantes a los encontrados por Reid (1983) para la misma especie en la cuenca del río Apure-Venezuela.

La actividad pesquera en la región Ucayali, como en toda al Amazonía, presenta una gran dispersión, es decir, se desarrolla en muchos lugares a lo largo de las principales cuencas de la región (Cañas, 2000). Contreras (1997) para un periodo de estudio de tres meses reporta a Centro Mashea, Utuquinia y San Antonio como zonas de pesca de doncella. Así mismo, Saavedra y Ferre (2001) reportan a Callería, San Antonio, Utuquinía, Aguas Negras, río Tamaya, Amaquiría y Mozote como zonas de su distribución. Los resultados del presente trabajo, no sólo confirman el hecho de que la actividad pesquera se desarrolla en muchos lugares a lo largo de las principales cuencas de la región, sino que el recurso estudiado presenta una distribución muy amplia, habitando: lagos, lagunas (cochas), tipishcas, quebradas y ríos, tanto del alto como del bajo Ucayali, que le permite desarrollarse sin verse afectada, hasta el momento. De las 74 zonas de pesca registradas, las principales zonas con potencial pesquero para esta especie por los volúmenes de extracción son Sheshea, Masisea, Aguas Negras y Santa Isabel, Pisqui, Aguaytía en alto y bajo Ucayali respectivamente.

Calcular la captura por unidad de esfuerzo (CPUE) constituye una de las medidas más útiles en la evaluación de recursos, ya que representa el índice de abundancia (Tresierra y Culquichicon 1995), pues relaciona la captura total, como peso total, de todas las especies capturadas con el esfuerzo total desplegado. Los resultados de este parámetro en el presente estudio, indican que la abundancia del recurso estudiado, en los primeros meses del año (que corresponden a la época final de lluvias) es baja, y en verano e inicios de la creciente el recurso es más abundante. Esto se debe precisamente a que en verano el nivel de agua ha disminuido haciendo más fácil la pesca. La abundancia de la doncella en la época de inicio de la creciente (últimos meses del año) se debe posiblemente a que en estas fechas el recurso migra para reproducirse, hecho muy conocido por los pescadores quienes aprovechan este comportamiento para realizar sus capturas. 


\section{CONCLUSIONES}

En líneas generales, la Pseudoplatystoma fasciatum, doncella, presenta hábitos alimentarios carnívoros, depredando 18 especies de peces, siendo las más frecuentes e importantes Prochilodus nigricans, boquichico, Pimelodus blochii, bagre, y Mylossoma duriventris, palometa, durante todo el año; con algunos cambios en su dieta principal como el Psectrogaster rutiloides, chío chío y Potamorhina altamazonica, llambina, en la época de la creciente.

El ciclo reproductivo para esta especie, empieza con la maduración sexual a finales de octubre hasta febrero, produciéndose enseguida el desove que finaliza en abril.

La talla media de madurez sexual para esta especie fue determinada en 93.92 y $78.49 \mathrm{~cm}$ de longitud total para hembras y machos respectivamente, siendo la talla de desove para hembras de 102.24 y para machos $87.89 \mathrm{~cm}$ de longitud total.

El comportamiento reproductivo de esta especie esta asociado al ciclo hidrológico del río Ucayali, coincidiendo la maduración sexual con la época de interfase a creciente y el desove con la época de creciente.

La producción pesquera de la doncella para el 2001 asciende a 1857.6 t, ubicando a este recurso en el segundo lugar después del boquichico. Los mayores desembarques provienen del alto Ucayali, siendo la zona de pesca más importante Sheshea.

\section{RECOMENDACIONES}

Es importante considerar los resultados obtenidos en este estudio para replantear la talla mínima de captura de la doncella que actualmente rige en el Plan de Ordenamiento Pesquero y asegurar una pesca sostenida a través del tiempo.

Por otro lado es necesario realizar estudios ecológicos para establecer las relaciones interespecíficas para la doncella a fin de predecir los cambios estructurales en poblaciones más explotadas, a fin de proponer medidas de regulación conjuntas.

\section{BIBLIOGRAFÍA}

BARTHEM, R.; H. GUERRA y M. VALDERRAMA. 1995. Diagnóstico de los recursos hidrobiológicos de la Amazonía. 2ª edición. Tratado de Cooperación Amazónica (TCA). Secretaría Pro Tempore. Lima-Perú.

CAÑAS, C. 2000. Evaluación de los recursos pesqueros en la provincia de Tambopata, Madre de Dios (julio 1995diciembre 1998). CI-Perú ediciones. Lima-Perú. 68 pp.

CONTRERAS, E. 1997. Biología reproductiva, hábitos alimenticios y pesquería de Pseudoplatystoma fasciatum (Linnaeus, 1776), doncella, en la Región Ucayali, de agosto a noviembre de 1997. Informe de prácticas preprofesionales para optar el título de biólogo perquero. Universidad Nacional de Trujillo. Perú.

GALVIS, G.; J. MUJICA y F. RODRÍGUEZ. 1989. Estudio ecológico de una laguna de desborde del río MeticaOrinoquia Colombiana. Centro Editorial Universidad Nacional de Colombia, Fondo Fen Colombia.

GARCÍA, A.; V. MONTREUIL y R. RODRÍGUEZ. 1998. Talla a la primera maduración y época de desove de Pseudoplatystoma fasciatum, doncella, en la Amazonía Peruana. IIAP. Iquitos-Perú.

GOULDING, M. 1980. The fishes and the forest, explorations in Amazonian Natural History. University of California Press, Berkeley. 280 pp. 
LAUZANNE, L.; G. LOUBENS y B. LEGUENNEC. 1990. Pesca y biología pesquera en el Mamoré medio (región de Trinidad, Bolivia). Interciencia. Nov-dic. Vol. 15 (6) : 452-460.

MONTREUIL, V.; A. GARCÍA y R. RODRÍGUEZ. 2001. Biología reproductiva de Prochilodus nigricans boquichico, en la Amazonía peruana. Folia Amazónica. Vol. 12 (1-2) : 5-13. IIAP. Iquitos-Perú.

NOVOA, D. y S. RAMOS. 1982. (Comp.). Los recursos pesqueros del río Orinoco y su explotación. Editorial Arte. Caracas-Venezuela. 358 pp.

REID, S. 1983. La biología de los bagres rayados: Pseudoplatystoma fasciatum y Pseudoplatystoma tigrinum, en la cuenca del río Apure, Venezuela. Revista UNELLEZ de Ciencia y Tecnología Producción Agrícola. Año 1, n. ${ }^{\circ} 1$ : 13-41. Barinas-Venezuela.

SAAVEDRA, J.C. y R. FERRE. 2001. Peces de importancia alimenticia en Ucayali. Boletín de Divulgación. Año I , n. ${ }^{\circ}$ 18. Ucayali-Perú.

SALINAS, Y.; E. AGUDELO y C. SÁNCHEZ. 1999. Peces de importancia económica en la cuenca amazónica colombiana. V Simposio Colombiano de Ictiología. Leticia-Amazonas-Colombia.

SMITH, N.J.H. 1981. Man, Fishes, and the Amazon. Columbia University Press, New York. 180 pp.

TRESIERRA, A. y Z. CULQUICHICÓN. 1993. Biología pesquera. Edit. La Libertad. Trujillo-Perú. 432 pp.

TRESIERRA, A. y Z. CULQUICHICÓN. 1995. Manual de biología pesquera. Edit. La Libertad. Trujillo-Perú. 227 pp.

TRESIERRA, A.; Z. CULQUICHICÓN y B. VENEROS. 2002. Biología reproductiva en peces. Edit. Nuevo Norte. Trujillo-Perú. 285 pp.

VAZZOLER,A. E. A. de M. 1981. Manual de métodos para estudos biológicos de populacoes de peixes: Reproducao e crescimento. CNPq. Programa Nacional de Zoología. Brasilia. 108 pp.

VAZZOLER, A. E. A. de M. 1996. Biologia da Reproducao de peixes Teleosteos: Teoría y Práctica. Apresentacao de Paulo de Torso de Cunha Chaves. Maringa: EDUEM. Sao Paulo: SBI. 169 pp.

WELCOMME, R.L. 1979. Fisheries ecology of floodplain rivers. Longman, London and N.Y. 317 pp. 